\title{
Modelling the Galaxy from survey data
}

\author{
A. C. Robin ${ }^{1}$, C. Reylé ${ }^{1}$ and D. Marshall ${ }^{1,2}$ \\ ${ }^{1}$ CNRS-UMR6213, Institut Utinam, Observatoire de Besançon, BP1615, F-25010 Besançon \\ cedex, France \\ email: annie.robin@obs-besancon.fr \\ ${ }^{2}$ Département de Physique, Génie Physique et Optique, Université Laval, Québec, QC, G1K \\ $7 \mathrm{P} 4$, Canada
}

\begin{abstract}
Recent optical and near-infrared surveys have considerably improved our knowledge of galactic structure and galactic evolution. Two ways can be used to infer this knowledge from datasets: either inversing the data to get parameters describing the Galaxy, or using a synthetic approaches to test scenarios of formation and theoretical models for star and galaxy formation and evolution, both approaches being complementary. Using the synthetic approach the Besancon Galaxy model allows to test scenarios for the structure and evolution of the Galaxy by comparing simulations with the survey data. Examples are given using the 2MASS survey. Future uses of astrometric survey data are shown to be able to efficiently constrain the kinematics and dynamics of the Galaxy.
\end{abstract}

Keywords. Galaxy: stellar content, Galaxy: evolution, Galaxy: kinematics and dynamics

\section{Introduction}

Constraints on Galactic structure and evolution come from a wide variety of data sets, such as multi-wavelength photometry, kinematics, microlensing events, among others. Scenarios of Galaxy formation and evolution are inferred from these constraints. An ultimate test of these scenarios can be done using these constraints piece by piece in order to build a population synthesis model which predictions can be directly compared with observations. This synthetic approach ensures that biases have been correctly taken into account and that the scenario is compatible with a variety of constraints. In recent years wide surveys have been obtained from optical and near-infrared photometry thanks to the wide-field CCD mosaic cameras, multi-object spectrometers, and the dedicated groundand space-based telescopes. Astrometric accuracy has been largely improved and catalogues with accurate proper motions are available, well-calibrated by Hipparcos and Tycho data. The homogeneity of these data sets is a great help for avoiding systematic bias percolating from one set to another, which have created difficulties in data interpretation in the past. All these data sets benefit to Galactic evolution studies and provide constraints on the population synthesis approach.

Here we report on the development of the Besançon Galaxy model based on this synthesis approach. We overview the basic scheme and input (Sect. 2) and we describe new results obtained by analysis of large data sets like the 2MASS surveys, in particular of the Galactic central region and on the disk external structure (Sect. 3). We then describe (Sect. 4) which constraints on kinematics have to be taken into account by any dynamical model and how the future astrometric surveys will help to build a fully self-consistent model of the Galaxy. 


\section{The population synthesis approach}

The population synthesis approach aims at assembling current scenarios of galaxy formation and evolution, theories of stellar formation and evolution, models of stellar atmospheres and dynamical constraints, in order to make a consistent picture explaining currently available observations of different types (photometry, astrometry, spectroscopy) at different wavelengths. The validity of a Galactic model is always questionable, as it describes a smooth Galaxy, while inhomogeneities exist, either in the disk or the halo. The issue is not to make a perfect model that reproduces the known Galaxy at all scales. Rather one aims to produce a useful tool to compute the probable stellar content of large data sets and therefore to test the usefulness of such data to answer a given question in relation to Galactic structure and evolution. Modelling is also an effective way to test alternative scenarios of galaxy formation and evolution.

The originality of the Besançon model, as compared to a few other population synthesis models presently available for the Galaxy, is in its dynamical self-consistency. The Boltzmann equation allows the scale height of an isothermal and relaxed population to be constrained by its velocity dispersion and the Galactic potential (Bienaymé et al. 1987). The use of this dynamical constraint eliminates a set of free parameters which are difficult to determine: the scale height of the thin disc at different ages. It gives the model an improved physical credibility. However this constraint is only applied at the solar position and perpendicular to the plane. A fully consistent dynamical model would apply self-consistency constraints at any position in the Galaxy.

The main scheme of the model is to reproduce the stellar content of the Galaxy, using some physical assumptions and a scenario of formation and evolution. We assume that stars belong to four main populations : the thin disk, the thick disk, the stellar halo (or spheroid), and the outer bulge. The modelling of each population is based on a set of evolutionary tracks, assumptions on density distributions, constrained either by dynamical considerations or by empirical data, and guided by a scenario of formation and evolution, that is to assume certain initial mass function (IMF) and star formation rate (SFR) for each population. More detailed descriptions on these constraints can be found in Haywood et al. (1997) for the thin disk, Reylé \& Robin (2001) for the thick disk, Robin et al. (2000) for the spheroid, and Picaud \& Robin (2004) for the outer bulge.

The Galactic model has been developed to return the results in the near-infrared and visible filters, but it has been extended to predict the stellar content in the X-ray domain (Guillout et al. 1996). More recently, the Hipparcos mission and large scale surveys in the optical and the near-infrared have led to new physical constraints improving our knowledge of the overall structure and evolution of the Galaxy. These new constraints are now included in the version of the model described in Robin et al. (2003).

\subsection{Limitations}

The model essentially produces a smooth Galaxy, which is certainly an over-simplification of reality. Inhomogeneities exist which cannot be easily modelled (clusters, associations, star forming regions). Major streams could be modelled, however, it might not be an objective of a model to reach such a degree of realism. To search for streams in observations, it may be more useful to produce simulations without streams and subtract them from the observations in order to amplify the contrast of the stream. A homogeneous model is also a good tool to estimate the degree of homogeneity present in real data with respect to the Poisson noise included in the smooth model. 


\section{2MASS survey}

The first effect visible in the star density distribution near the Galactic plane is due to the dust, even at near-infrared wavelength. Without a good estimate of the extinction and of its distance it is nearly impossible to understand the structure in the disk. Hence we have first attempted to build a 3D model of extinction in the plane. The 2MASS survey allows to study large scale structures in the Galaxy, particularly in the Galactic plane because the NIR data are well suited to study stellar populations in regions of medium to high extinction. In this survey the star counts appear to be dominated by red clump giants. Here we show how it can be used to determine the $3 \mathrm{D}$ distribution of the extinction and then to constrain structure of the disk.

\subsection{Extinction model}

Extinction is so clumpy that it drives the number density of stars more than any other large scale stellar structure. However, photometry and star counts contain information about the dust extinction. Marshall et al. (2006) have shown that the 3D extinction distribution can be inferred from the stellar colour distributions from the 2MASS survey. Using stellar colours in J-K as extinction indicators and assuming that most of the model prediction deviations in the observed colours arise from the variation of extinction along the line of sight, they built a $3 \mathrm{D}$ extinction model of the galactic plane $(-10<b<10 \mathrm{deg}$ and $-90<l<90)$. The resolution in longitude and latitude is 15 arcmin and the resolution in distance varies between $100 \mathrm{pc}$ to $1 \mathrm{kpc}$, depending on stellar density and the density of dust along the line of sight. The resulting 3D extinction model provides an accurate description of the large scale structure in the disk of dust.

\subsection{Comparing 2MASS with model star counts}

A comparison between the basic model described in Robin et al. (2003) - modified by the 3D extinction model of Marshall et al. (2006) - and 2MASS star counts (Fig. 1) shows that the model reproduces the data with a high degree of realism in the plane, even without modeling the spiral arms. It means that either the spiral structure does not have a high contrast or that the 2MASS data are not a good tracer for these arms. This is expected as the model estimates that the 2MASS counts are dominated by red clump giants which are old stars $(90 \%$ have ages $>1$ Gyr, having made at least 4 revolutions around the Galaxy) and have lost the memory of their place of birth. However, two non-axisymmetric features are not well modelled : the external bulge and the warp. This is easily seen in Fig. 2. The triaxial bulge model has been adjusted by Picaud \& Robin (2004) in the central region limited in longitude from -10 to +10 and -4 and +4 in latitude using, the DENIS survey data (Epchtein et al. 1997). Comparisons between predictions and 2MASS data in Fig. 2 show that this region is well reproduced but the more external bulge is not : differences appear at latitudes $4<|b|<10$ and at longitudes $5<|l|<10$. We are presently investigating this problem. Our preliminary conclusion is that the central part of the Galaxy $(-5<l<5)$ is well fitted by the triaxial old bulge model with a small angle between the major axis and the sun-center axis, as found by Picaud \& Robin (2004), while the external part in the plane $5<|l|<10$ is better fitted by a bar with a higher angle, the bar being also a more elongated structure than the bulge with a smaller scale height of its minor axis.

\subsection{The Galactic warp}

Warps may have originated from interactions between the disk and (i) the dark halo (if the angular momenta are not aligned), (ii) nearby satellite galaxies, such as the Sgr dwarf 


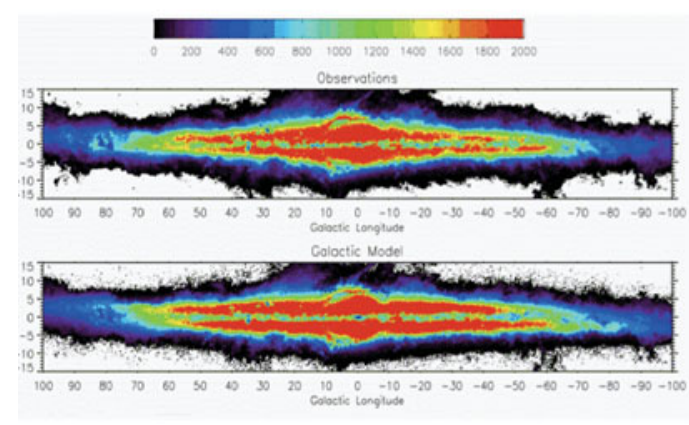

Figure 1. 2MASS star counts per square degree and model simulations in the Galactic plane as a function of longitude and latitude. The scale is number of stars per square degree. Top: Data, Bottom: Model.

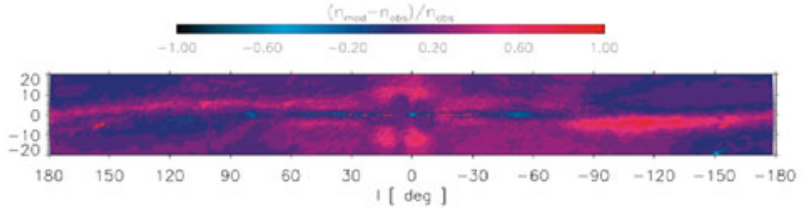

Figure 2. Relative difference between 2MASS star counts per square degree and standard model simulations in the Galactic plane (simulated minus observed, divided by observed star counts).

or the Magellanic Clouds, (iii) infalling intergalactic gas. Details of these scenarios can be found in Bailin (2003), Garcia-Ruiz (2002) or López-Corredoira et al. (2002).

For simulations we use a tilted ring model such as by Porcel et al. (1997). The free parameters are the galactocentric radius where the warp starts, the slope and the azimuthal angle of the line of nodes. Following Burton (1988), we assume that the Sun lies approximately on the line of nodes of the warp $\left(\theta_{\max }=90^{\circ}\right)$. We adopt a slope similar to Gyuk et al. (1999) $\left(\gamma_{\text {warp }}=0.18\right)$. Preliminary results obtained from the analysis of the DENIS survey (Derrière \& Robin 2001; Derrière 2001) indicate the best value for a starting galactocentric radius of the warp to be at $R_{\mathrm{warp}}=8.4 \mathrm{kpc}$, close to $R_{\odot}$.

It appears from Fig. 2 that the slope of the warp is not correct in model simulations. Apparently, the adopted slope is too high. Using a value of 0.09 gives very good agreement between the data and simulations at positive longitudes (see Fig. 3), while at negative longitudes that is still unsatisfactory. This effect seems to be independent of the assumed slope, or the disk scale length (assumed to be $2.2 \mathrm{kpc}$ ), or to the sun-center distance (assumed to be $7.9 \mathrm{kpc}$ ). Alternative models of the warp could explain this structure. It is well known that the HI warp is not symmetrical : the gas warp bends back to the Galactic plane in the southern hemisphere at $\mathrm{R}>15 \mathrm{kpc}$ (Burton 1988). The stellar warp may also follow this feature. Tweaking the model may allow for the adjustment of this feature. However, a dynamical approach is needed to constrain the origin of this warp and its link to the gaseous warp.

\section{Kinematics and dynamics of the Galactic stellar populations}

The objective of the construction of this model of stellar population is to have a totally self-consistent and realistic model of the Galaxy, consistent with our knowledge 

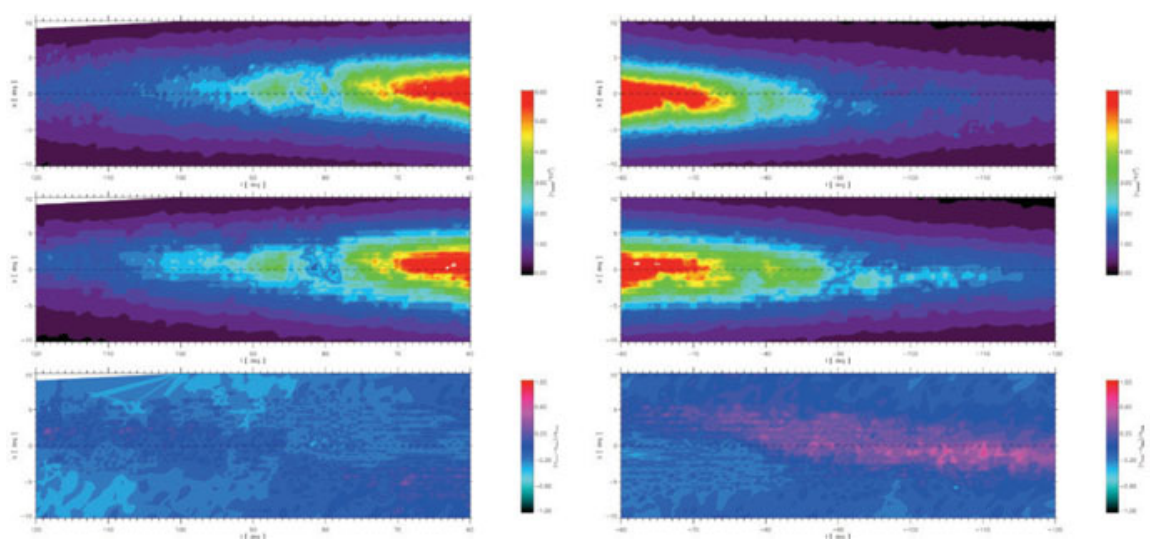

Figure 3. Comparison of 2MASS star counts (top panel) with modeled ones (middle panel), and relative difference between the two (bottom panel) for the warped region at positive longitudes (on the left) and at negative longitudes (on the right), with a warp slope of 0.09.

of Galactic evolution, including chemical and dynamical aspects. However, Galactic dynamics studies are complex and require the modelling of some kinematical features of stellar populations such as invisible matter, non-axisymmetries, inhomogeneities. We do not discuss here the kinematical and dynamical features in the halo, nor the thick disk, which are detailed in Majewski (2008) and Haywood (2008) at this conference. Instead, we concentrate on the disk kinematical features and their relation with dynamics.

\subsection{Disk non-axisymmetries}

The stellar disk shows a number of kinematical features related to its axisymmetries : the stars rotate in the disk with a nearly flat rotation curve in a large part of the disk. The stellar kinematics can be described as a velocity ellipsoid $\left(\sigma_{U}, \sigma_{V}, \sigma_{W}\right)$. It has been shown that the values of velocity dispersion follows the stellar age : old stars have larger velocity dispersions than the young ones. That is generally explained by the orbit diffusion due to interactions with the gaseous disk (spiral structure and giant molecular clouds). The vertical velocity dispersion runs from about $5 \mathrm{~km} / \mathrm{s}$ for young stars (this is about the velocity dispersion from the gas they were born) to a range of 15 to $30 \mathrm{~km} / \mathrm{s}$ for the oldest disk stars (about $10 \mathrm{Gyr}$ ). There are large discrepancies in the literature for the latter values. Gómez et al. (1997) find a maximum value of $17 \mathrm{~km} / \mathrm{s}$, like Famaey et al. (2005), while Rocha-Pinto et al. (2004) argue for $30 \mathrm{~km} / \mathrm{s}$ and Nordstrom et al. (2004) find $25 \mathrm{~km} / \mathrm{s}$. This disagreement may be explained by contamination of the oldest disk star samples by thick disk stars, which are not easily distinguished from the old thin disk neither by their abundances nor by their rotational velocity (Haywood 2008). Hence, the efficiency of the orbit diffusion in the disk is still a matter of debate, as well as the physical processes at work.

On the top of these axisymmetric features, non-axisymmetries are distinguishable even at the solar position, coming from the effects of bar (Fux 2001; Dehnen 2000) or from the spiral structure (Famaey et al. 2005). These non-axisymmetries translate into observables in the form of non zero mean motion of the Local Standard of Rest towards the Galactic center, or away from the Galactic Plane, or by a vertex deviation of the velocity ellipsoid. This last feature, long known from local kinematical studies, may also depend on the ages of a star sample considered. 


\subsection{Disk inhomogeneities}

Disk inhomogeneities in positions are also well known. Clusters and associations are numerous in the disk but they participate to the mass distribution to a small level and do not perturb much the whole dynamics of the system. On the contrary, there exist inhomogeneities in the phase space which are very interesting to study as they inform on the overall dynamics. Famaey et al. (2005), following an earlier study of Eggen (1958) have identified a number of streams in the local neighborhood from the Hipparcos catalogue. From the analysis of $3 \mathrm{D}$ velocities of $\mathrm{K}$ and $\mathrm{M}$ giants they identify up to 6 kinematical groups and among them, the Hercules stream, Hyades supercluster, Sirius Moving group and the Pleiades supercluster. They show that these groups are not homogeneous in age. Their common motions cannot originate from a common birth history. Hence, the most probable explanation is that they are due to dynamical perturbation by transient spiral waves or related to the bar's outer Lindblad resonance.

The vertex deviation gives another way to investigate the non-axisymmetry in the disk. Soubiran et al. (2003) investigated the vertex deviation from a non local sample of stars towards the North Galactic Pole. They found no evidence of vertex deviation for old disk stars at different distances from the Galactic plane, showing that this population looks well relaxed and non perturbed. This is clear disagreement with Famaey et al. (2005) conclusions which argue that the vertex deviation is noticeable even for old populations and probably related to the Hercules stream. The complex dynamics of the thin disk is yet to be understood.

\section{Implications of large scale astrometric surveys}

Present and future large scale astrometric surveys will allow us to take a big step towards the understanding of the Galaxy formation and evolution. The Galactic potential and Galactic dynamical features are difficult to constrain when one has only 1 or 2 velocity vectors (radial velocities or proper motions) and 2 out of 3 accurate positions, the distances being very inaccurate in the absence of trigonometric parallaxes. There have been significant attempts to produce dynamical models since the Hipparcos mission, and several surveys of radial velocities and proper motions have been completed. However, the determination of a unique dynamical model is nearly impossible and the solutions remain degenerate in the absence of good distances. This is particularly true for the bulge, where different models exist with quite different dynamical properties. This will change when true $6 \mathrm{D}$ phase space will be available, for example from the Gaia space mission. Gaia will supply us with very accurate parallactic distances (with an accuracy of about $10 \%$ at the Galactic center and much better in the solar neighbourhood), very accurate proper motions and reasonably good radial velocities. It could be complemented by the groundbased radial velocity measurements. RAVE (RAdial Velocity Experiment) (Steinmetz et al. 2006) has already started a survey of a significant part of the sky with 1 million stars to be measured from medium resolution spectroscopy giving the radial velocity with an accuracy of about $2 \mathrm{~km} / \mathrm{s}$ together with stellar atmosphere parameters (effective temperature, metallicity and gravity). This survey is cross-identified with ground based proper motions surveys giving access to at least 5D phase space data (accurate distances are still missing). This will furnish very good data towards the construction of a realistic dynamical model of the Galaxy, but still be limited to a region of a few kiloparsecs around the sun. They are some attempts to deduce such a model by inversion of the parameter space (Veltz et al. 2007). Even if this study has not given firm conclusions because it is limited by the sample size, it shows that this approach is feasible and could lead to 
a unique solution if the data are accurate enough and the sample is large enough. This kind of analysis is expected to be much more useful during the analysis of multivariate data from the Gaia mission.

\section{Conclusions}

Population synthesis models are important tools for interpretation of data coming from large scale surveys, either photometric, astrometric or both. Although imperfect, they allow to examinate the distribution functions corresponding to a given scenario, by a way of producing simulations to be directly compared with the data sample. Self consistent dynamical models coupled with population synthesis (which reproduce different aspects of the data and can account for the photometric selection and observational bias) are somehow lacking. Large efforts to produce complete chemo-dynamical models have already been started by different groups and need to be continued. Future astrometry missions will need these models to ease their interpretation and to help to construct more realistic scenarios for Galactic formation and evolution.

\section{References}

Bailin, J., 2003, ApJ, 583, L79.

Burton, W. B.: 1988, Galactic and Extragalactic Radio Astronomy, 2nd version, p. 295, SpringerVerlag

Bienaymé, O., Robin, A. C., \& Crézé, M. 1987, A\&A, 180, 94

Cutri, R. M., Skrutskie, M. F., van Dyk, S. et al., 2003, Explanatory Supplement to the 2MASS All Sky Data Release

Dehnen W., 2000, AJ, 119, 800

Derrière, S. \& Robin, A. C. 2001, ASP Conf. Ser. 232: The New Era of Wide Field Astronomy, p. 229

Derrière, S. 2001, Thèse de doctorat, Université Louis Pasteur, Strasbourg.

Eggen O. J., 1958, MNRAS, 118, 65

Epchtein, N. et al. 1997, The Messenger, 87, 27

Famaey B., Jorissen A., Luri X., et al., 2005, A\&A, 430, 165

Fux R., 2001, A\&A, 373, 511

Garcia-Ruiz, I., Kuijken, K., Dubinski, J., 2002, MNRAS, 337, 459

Gómez, A. E., Grenier, S., Udry, S., Haywood, M., Meillon, L., Sabas, V., Sellier, A., \& Morin, D. 1997, ESA SP-402: Hipparcos - Venice '97, 402, 621

Guillout, P., Haywood, M., Motch, C., \& Robin, A. C. 1996, A\&A, 316, 89

Gyuk, G., Flynn, C., \& Evans, N. W. 1999, ApJ, 521, 190

Haywood, M., Robin, A. C., \& Crézé, M. 1997, A\&A, 320, 440

Haywood M., 2008, in this volume p.458

López-Corredoira, M., Betancort-Rijo, J., Beckman, J. E., 2002, A\&A, 386, 169

Majewski S., 2008, in this volume p.450

Marshall, D. J., Robin, A. C., Reylé, C., Schultheis, M., \& Picaud, S. 2006, A\&A 453, 635

Nordstrom B., Mayor M., Andersen J., et al., 2004, A\&A, 418, 989

Picaud, S., \& Robin, A. C. 2004, A\&A, 428, 891

Porcel, C., Battaner, E., \& Jimenez-Vicente, J.: 1997, A\&A 322, 103

Reylé, C. \& Robin, A. C. 2001, A\&A, 373, 886

Robin, A. C., Reylé, C., Crézé, M. 2000, A\&A 359, 103

Robin, A. C., Reylé, C., Derrière, S., \& Picaud S. 2003, A\&A, 409, 523.

Rocha-Pinto, H. J., et al., 2004, A\&A, 423, 517

Soubiran, C., Bienaymé, O., \& Siebert, A., 2003, A\&A, 398, 141

Steinmetz, M. et al., 2006, AJ 132, 1645

Veltz, L. et al., 2007, submitted to A\&A 